\title{
Integrating referral to community-based cancer information and support services in a hospital setting
}

\section{David JT. Marco}

Cancer Council Victoria, 615 St Kilda Rd, Melbourne VIC 3004 Australia \& School of Psychological Sciences, University of Melbourne, Victoria, Australia. dmarco@unimelb.edu.au

\author{
Anna G. Boltong \\ Cancer Council Victoria, 615 St Kilda Rd, Melbourne VIC 3004 Australia \& \\ School of Health Sciences, University of Melbourne, Victoria, Australia. \\ anna.boltong@cancervic.org.au
}

\section{Adrian Dabscheck}

Western Health, Sunshine Hospital, Furlong Rd, Footscray VIC 3011 Australia. adrian.dabscheck@wh.org.au

\section{Georgina Akers}

Department of Health and Human Services, 50 Lonsdale St, Melbourne VIC 3000, Australia. georgina.akers@dhhs.vic.gov.au

\section{Michelle Pryce}

Gippsland Regional Integrated Cancer Services, Latrobe Regional Hospital, 10 Village Ave, Traralgon West VIC 3844, Australia.

mpryce@Irh.com.au

\section{Victoria M. White}

Cancer Council Victoria, 615 St Kilda Rd, Melbourne VIC 3004 Australia \&

School of Psychology, Deakin University, Victoria, Australia.

vicki.white@cancervic.org.au

Corresponding author: Prof Victoria White

vicki.white@cancervic.org.au

+61395146352

Word count: 3988 words (excluding abstract, tables and figure captions).

\section{Acknowledgements}

We would like to acknowledge the support and involvement from Western Health and Gippsland Cancer Care Centre staff and patients who participated in this study. 


\section{Abstract}

\section{Purpose}

To implement and evaluate a hospital-based referral mechanism to increase patient uptake of community-based cancer information and support services. Feasibility and acceptability of the intervention and education program was evaluated. Changes in usage of Cancer Council Victoria's cancer information and support telephone line were investigated.

\section{Methods}

A 6-month study was conducted in one metropolitan and one regional cancer treatment hospital. Clinicians attended an education session regarding referral mechanisms to Cancer Council support services. Clinicians completed a questionnaire, and consenting patients participated in a semistructed telephone interview for the project evaluation. The number of calls made from patients at study sites was monitored.

\section{Results}

Fifty-two clinicians were trained and referred a total of 430 patients to the cancer information and support service during the study period. Calls from patients increased by up to $100 \%$ per month from baseline following the implementation of the referral mechanism. Staff evaluations showed support for the referral mechanism and its incorporation into routine practice. Interviews were conducted with 45 patients; most remembered receiving the referral and were positive towards the intervention.

Common reasons patients gave for not acting on the referral included forgetting, lack of need, timing, and burden of information.

\section{Conclusions}

There is preliminary evidence that this intervention increases awareness and uptake of communitybased cancer information and support services. Ongoing clinician education and improvements in patient-clinician communication are important for effective translation from referral to service uptake. Consideration of the most appropriate time in a patients' care trajectory to introduce a referral is warranted.

Keywords: cancer, intervention, referral, support services, hospital, information 


\section{Background}

Information about cancer, its treatment, possible long and short term effects, the health system and availability of supportive care services plays an important role in enhancing a patient's ability to cope, initiate self-care behaviours, and maintain their quality of life[1-5]. Addressing information needs and the psychosocial consequences of cancer is recognised as an integral component of optimal care[6].

Guidelines recommend that all patients and their families receive comprehensive information about their disease, side effects and treatment options[6]. However, many patients still report psychological/emotional, informational, practical, and sexuality unmet needs[7,8]. In many countries community-based cancer societies provide information, emotional and practical support services to patients and their families. These, often charity-based societies, sit alongside the health system producing resources for use within treatment centres while also providing services that can be accessed independently of the health system. Many societies have developed telephone-based cancer information and support (CIS) services to address the needs of patients (e.g. $[9,10])$. These services, staffed by cancer nurses in Victoria, Australia, offer a mechanism for providing information, support and referrals to psychosocial interventions for patients and families throughout treatment and survivorship[11,12]. However, many people affected by cancer are unaware of these services[13,14]. Promoting awareness and use of CIS services may increase patients and their family or support network's options for accessing support and information.

Clinician referral or recommendation encourages patient use of CIS services[14]. An Australian study found the likelihood of using written and web-based CIS services increased nearly 10 times, and using psychosocial services increased nearly 12 times following referral from a clinician[13]. However, few clinicians regularly refer patients to CIS services[13,14]. An Australian study found that less than $10 \%$ of clinicians who were aware of the Cancer Council's support service regularly referred patients to this service[15]. Poor knowledge about services, lack of time and resources to investigate and refer to services, and negative perceptions of supportive care interventions are common barriers to clinician referral in the oncology context[16].

Several studies have attempted to improve clinician referral to CIS services. An early study examined the impact of clinicians handing a CIS promotional brochure to patients, finding that $4 \%$ of patients called a CIS service when simply handed an information brochure[17]. A subsequent study involved patients receiving a clinical recommendation and specific referral to receive either four or one outcalls from CIS[18]. In the control condition, patients received a clinician referral (recommendation and referral slip) to CIS, but initiated contact themselves. In this condition, $18 \%$ of patients called the service[18]. The higher proportion of patients contacting the service in this condition compared with the earlier study, may demonstrate the influence of a specific referral to the service.

Increasing clinicians' knowledge and positive perceptions of CIS services and providing referral strategies may increase clinician referral to these services. Drawing on the literature regarding changing provider behaviours[19-21], we developed a method for integrating clinician referral to Cancer Council Victoria's Cancer Information and Support Service. We incorporated an educational outreach training program[22] and engaged local clinical champions to support the project's implementation and assist in addressing local barriers. As referring patients to other services or prescribing drugs is considered usual practice for clinicians, we developed a referral slip to refer patients to the information and support service. In this paper, we report the development and integration of this referral mechanism into clinical practice. We also present results evaluating the feasibility, acceptability and impact of the intervention. 


\section{Methods}

\section{Design and setting}

Figure 1 shows an overview of the study design. The study was conducted in five clinical units spanning two hospital sites comprising day oncology, palliative care, lung outpatients, and radiotherapy. The intervention ran for a six-month period in each unit.

\section{$<$ FIGURE 1 ABOUT HERE>}

\section{Designing the intervention}

\section{The Cancer Information and Support Service}

Cancer Council Victoria's CIS service provides a telephone support line as the main referral pathway into this service. The service is staffed by experienced oncology nurses who are trained to provide evidence-based information tailored to the patient's needs. The service is underpinned by a database of community and hospital services allowing staff to refer patients to relevant services at their hospital (e.g. social work services) or within their local community (e.g. home help services). Services delivered by Cancer Council Victoria may also be referred to and include peer support, counselling, free legal and financial planning programs, and information resources. This service provides a mechanism for addressing information and support needs of all cancer patients, overcoming barriers of geography, language (an interpreting service is available) and literacy. A systematic review has shown high user satisfaction with Cancer CIS services[23], with a recent study finding that service use can reduce worry, extend support avenues and develop new knowledge and language to assist interactions with healthcare teams[10].

\section{Engagement with clinical champions and teams}

An onsite hospital team comprising administration, research, and clinical staff was established at each site to support the project's implementation. Clinical champions were identified to promote and support the project onsite, facilitate staff engagement and ensure the project's design was compatible with hospital practices and local patient needs. Champions had strong links to clinical staff, were engaged in research, and were supporters of CIS. Researchers and the onsite committees met three times to arrange the study's design and implementation. Heads of participating clinical units were present at meetings and involved in implementation discussions.

\section{Referral pad and educational resource}

The study utilised a prescription pad-style referral form comprising a checkbox list of CIS services. The programs and services listed were determined in consultation with site clinicians, with priority given to those CIS services not available at the hospital. On request from the clinical committee at one site, CIS contact information was translated into the five languages most commonly spoken by the hospital's patients and printed on the back of the referral form. Clinicians and consumers reviewed the referral form's design and readability. .

An educational resource was designed to accompany the referral pad and act as an information resource for clinicians. The guide provided service details including access procedures and eligibility criteria.

\section{Health care provider education sessions}

Health care providers attended a single 50-minute education session delivered by the research team and CIS staff. The session was divided into two sections. The first section comprised a presentation with handouts, providing information about CIS and available services. The second section involved disseminating the study resources, discussion of the study design and use of the referral form. Opportunities for questions and discussion were provided throughout the session. 
Education sessions were scheduled based on clinical staff availability with champions working with clinical teams to identify two times when staff would be available to attend sessions. All staff from each participating unit were encouraged to attend the sessions. Lunch was provided if sessions occurred over a lunch break. The multiple education sessions aimed to accommodate part-time staff and the need for some staff to remain in the clinic during the session. The number of staff not attending the information session was not recorded.

Due to clinical demands, some staff could not attend the onsite education session. Instead, onsite champions delivered one-on-one education sessions to these clinicians following an academic detailing model[24,25]. These sessions were undertaken at a time suitable to the clinician, which may have been a lunch break or in clinic slots when patients failed to attend. Champions tailored the education session to accommodate the time available. .

\section{Referral procedure}

If, during their normal clinical interactions, clinicians identified patients who might benefit from a CIS service, they were asked to tick the corresponding service on the referral form, hand the referral to the patient and encourage them to call CIS to access the service. The message from the clinician to the patient was brief yet specific, with clinicians tailoring the referral to the need of the patient at that time. No formal script was provided to clinicians. Rather, they were asked to introduce the referral at an appropriate time, respecting the patient's current condition and level of understanding or comprehension of the information. Non-English speaking patients were referred to the free interpreter phone service that would connect callers with CIS in their language. Staff collated referral carbon copies at a central location, usually the nurses' station or reception desk. Champions collected the referral copies and posted them to the research team. Contact details of patients agreeing to participate in an evaluation interview were provided through the copies.

\section{Evaluation}

\section{Health care providers}

Clinicians attending the education session completed an evaluation survey to gauge acceptability, prior awareness of CIS and perceived limitations of the intervention. At the end of the study, clinicians completed another survey assessing their use and acceptability of the referral mechanism,,knowledge of CIS, perceptions of the intervention's usefulness, impact of referral on time and resources. Onsite champions distributed the post-study surveys. Free-text questions allowed clinicians to raise any concerns or recommendations relating to the intervention and its sustainability.

\section{Patients}

As part of the referral process, patients were asked for their consent to be contacted by the research team for evaluation purposes. To allow patients time to act on the referral, consenting patients were contacted approximately one month following receipt of their consent. Prior to patient contact, hospital staff reviewed the list of consenting patients to exclude those known to be too ill to participate or who were deceased. Those remaining on the list were contacted by telephone and asked consent to participate in a semi-structured telephone interview obtained. Interviews were typically 10-15 minutes long, depending on patient recall regarding the referral. The interviewer worked through a list of questions regarding experience receiving the referral, response to the referral, any actions resulting from the referral, and reasons for not acting on the referral. Questions were asked in an order that seemed natural to the conversation with the patient, with the interviewer ensuring all relevant questions were asked.

\section{Impact on calls to CIS}

As per their standard protocol, all calls to CIS were logged into a database with mandatory demographic and treating hospital information fields. The number of calls from each study site in the 
three months prior to the intervention period (baseline) and the number for each month of the intervention period were derived from database records. There were no active public relation campaigns promoting CIS services during the study period that could potentially affect the number of calls made to the service.

\section{Data analysis}

Descriptive statistics (means, frequencies) were used for clinician evaluation survey responses, the number of referrals distributed and total number of services referred. Independent samples t-tests were used to investigate any differences in clinician scores between hospital sites for the education session evaluation. The number of calls to CIS from patients treated at each study site and overall CIS calls received were extracted for the baseline and intervention months. Regression lines were calculated to identify trend differences between baseline and interventions months for each site. A one-sided independent samples t-test was conducted for each site to investigate whether average monthly calls were greater during the intervention than baseline period. Services on the referral were grouped into four main categories ('information', 'practical services', 'support groups', and 'additional free services'). Practical services included access to professional advice on employment, financial and legal matters. Free services were those hosted by Cancer Council Victoria and included wigs, phone-based psychosexual counselling, and exercise and nutrition coaching over the phone. Chi square tests were used to determine whether the proportion of patients referred to the different service categories differed between hospital sites. Interview responses relating to reasons for not acting on the referral were reviewed and themes identified. Response frequencies were calculated for questions regarding content recollection, comprehension, use of CCV service, and satisfaction.

\section{Ethics and consent}

The study was approved by ethics committees associated with Cancer Council Victoria and each study site. A waiver of individual patient consent was granted, enabling all patients in participating units to be included as trial participants.

\section{Results}

\section{Health care provider education sessions}

Across the two sites, 52 clinicians comprising nurses, social workers, radiation therapists, and medical oncologists attended the education sessions. However, only 40 clinicians complete the education evaluation survey ( $77 \%$ response rate). An additional 14 clinicians were trained individually by onsite champions but did not complete the evaluation. No clinicians refused participation in the study.

Results in Table 1 suggest there was a high level of satisfaction with the education sessions, with staff reporting that the content was interesting, useful, and understandable. The most common written feedback illustrated a lack of awareness of CIS programs prior to the education session, and the value of being informed of the services available $(\mathrm{N}=22)$. There were no significant differences in evaluation scores between hospital sites (all $\mathrm{p}>\mathrm{.05}$ ).

\section{<TABLE 1 ABOUT HERE>}

\section{Referrals}

Across the two hospitals, clinicians referred a total of 430 patients to CIS. Of the 430 referral slips administered, $274(64 \%)$ referral forms had no services selected, $36(8 \%)$ had one service selected, with the remaining $120(28 \%)$ forms having more than one service selected. Of those patients that had at least one service selected on the form, an average of 4 services were recommended $(M=4.1$, $\mathrm{SD}=2.4)$. The most commonly referred services were 'emotional and practical support over the phone' 
$(\mathrm{N}=57)$, 'information about cancer and treatment' and 'local face-to-face support groups' (both $\mathrm{N}=55$ ). The type of referrals offered to patients was shown to differ significantly between hospital site $\left(X^{2}(3)=14.8, p<.01\right)$ with 'support group' services being more common $(36 \%)$ and 'additional free services' being less common (15\%) at the regional site than the metropolitan site (24\%, $24 \%$ respectively).

\section{Impact on calls to CIS}

Figure 2 shows call volume to CIS for each site compared to total calls made to CIS.

\section{$<$ FIGURE 2 ABOUT HERE>}

\section{Metropolitan site}

A seasonal drop in calls due to the 2014 Christmas period is visible for both the intervention site and total calls to CIS (Figure 2). Baseline data from October 2014 was used for comparison as it better reflected normal call volume (Jan-Feb 2015) and was not affected by the seasonal drop.

Monthly calls from patients attending the metropolitan intervention hospital increased from 12 in October 2014 to a maximum of 24 in July 2015 (100\% increase). This was a significant increase from the number of calls received during baseline months $(t(9.5)=1.90, p<.05)$. During May 2015, one of the participating units was physically relocated, affecting 19 (54\%) of the staff involved in the study at this site. A one-month "blackout" period was introduced, suspending referrals from mid-April to midMay 2015 to allow staff time to adjust to the move. The large drop in calls in August 2015 coincided with the termination of the intervention in the two largest participating units at the site. Therefore, results from August-September 2015 are reflective of only one unit continuing with the intervention at this site.

\section{Regional site}

As expected, when compared to the metropolitan site, there were fewer inbound calls from the regional site, reflecting its smaller size. However, the impact of the intervention on calls to CIS was similar with a significant two-fold increase in call volume when comparing monthly call averages at baseline $(M=2.3, S D=1.5)$ with those for the intervention period $(M=4.7, S D=2.1),(t(5.3)=2.02, p<$ $.05)$.

\section{Acceptability}

\section{Health Care Providers}

Post-intervention surveys were completed by 40 clinicians ( $61 \%$ response rate). Overall, most clinicians reported the intervention was acceptable and feasible (Table 2).

\section{<TABLE 2 ABOUT HERE>}

Common barriers reported by clinicians included patient refusal or resistance to receiving the referral $(\mathrm{N}=5)$, patient's issues already being addressed $(\mathrm{N}=2)$, and time constraints limiting ability to address patient's issues $(\mathrm{N}=2)$. Clinician recommendations for improvement included ongoing staff education and reminders $(\mathrm{N}=4)$, mechanisms to motivate use of the referral pad $(\mathrm{N}=4)$, more referral pads to be accessible to staff $(\mathrm{N}=2)$, and focus referral in outpatient settings where little support is available $(\mathrm{N}=1)$.

\section{Patients}

Of the 147 patients (34\%) consenting to be contacted, 76 were approved for contact by study site staff. Forty-five patients were interviewed ( $59 \%$ of the 76$)$. The remaining patients declined participation $(\mathrm{N}=4)$, were deceased $(\mathrm{N}=4)$ or could not be contacted $(\mathrm{N}=23)$.

Thirty-two patients could recall the referral. Twenty-nine (64\%) patients understood the purpose of the referral and the process for contacting CIS. Only 17 patients could recall the detail of what the 
clinician said to them when receiving the referral. No-one reported feeling pressured to call CIS. Four patients interviewed $(9 \%)$ reported contacting CIS and all were very satisfied with the service received.

Forgetting about the referral and not feeling a need for the service were the two most common reasons for not contacting CIS (Table 3).

\section{$<$ TABLE 3 ABOUT HERE>}

\section{Discussion}

In an effort to increase provision of information and support to people affected by cancer, this study examined the feasibility of integrating a clinician referral to a community-based cancer information and support service into usual clinical care. Drawing on existing literature regarding changing provider behaviours[19-21], the study used an educational outreach paradigm to inform clinicians about CIS services and utilised a referral slip to encourage referral of patients to the service. We found that both the education session and the referral procedure were acceptable to clinicians and patients. Our study also found that the number of calls to CIS from our intervention sites increased during the intervention period. Importantly, we showed that implementation of the intervention was feasible and effective in both a large metropolitan hospital and regional hospital.

Engagement from clinical champions was important in delivering the training program and implementing and maintaining use of the referral mechanism during the intervention period. Onsite champions ensured communication between the research and clinical units remained open, especially during periods of staff turnover and department restructure. This is in line with previous research suggesting that champions have an important role in promptly providing solutions to problems arising, thereby minimising any negative impact on clinician engagement[26].

The referral mechanism used was designed to ensure that services meeting the patient's needs could be recommended. However, more than half the referral forms given to patients had no services selected. While feedback from some clinicians suggested this was due, to time constraints, other clinicians indicated that they intentionally did not select specific services in an attempt to inform patients of the full suite of CIS services, understanding that patients' needs may change throughout their cancer care and certain services become more salient at another time.

While calls to CIS from the study sites were too few to infer any strong statistical conclusions, both sites showed an increasing trend in calls to CIS during the intervention period. This increase was made at a time when there was an overall decline in total calls to CIS $(-27 \%)$, suggesting the intervention may have helped to counter this decline at the study sites. While not conclusive, the decline in state-wide call volume to the service during the study period supports the suggestion that there were no effective CIS public relation campaigns that could be driving the increase in call volume from the study sites.

Only four of the 45 patients interviewed called CIS in response to the referral. Patients reported several barriers to their use of the service, with the main barriers relating to lack of need and poor timing, often because patients felt overloaded with information. At the end of the study, discussions with clinical teams identified 'transition points' in patient care which might provide appropriate opportunities for patient referral, including the transition from one treatment to another, or the end of active treatment when hospital support services are no longer readily available. However, as only a proportion of cancer patients prefer to use a telephone service to seek CIS support, services need to offer multiple avenues for accessing support including internet-based services.

While the education session evaluations suggested that many clinicians did not have a good understanding of the services CIS delivers, clinicians were familiar with the Cancer Council brand and considered it a trustworthy organisation. Subsequent discussions with clinicians in this study 
suggested that concern about the type and quality of support offered to their patients was a barrier to referring patients to services. This suggests there may be greater resistance to referring patients to services delivered by new or unfamiliar organisations. Developing collaborative relationships with the onsite teams in the early phase of the project may be important in future studies as it will help to ensure clinician trust in the organisation delivering support services.

Clinicians relayed the need for ongoing education and availability of referral resources including mechanisms to motivate use of the referral. Ongoing clinician education has been shown to be important in successfully changing behaviour[13,14,16,22]. An ongoing system of education comprising onsite visits from CIS nurses and electronic distribution of, and access to, CIS resources may assist in keeping clinical staff informed and engaged with available support services. Introducing flexible training options may be necessary for an intervention of this type in a larger rollout. Having tailored education sessions that can adapt to the time clinicians have available could provide further opportunities to train more staff (e.g. during various team meetings). Onsite champions could facilitate this process and inform CIS of the frequency at which this information is required with respect to staff volume, turnover and the unmet needs of their patients. Harnessing champions' site-specific knowledge and existing relationships with clinical teams could facilitate a larger, multi-centre rollout of this intervention.

We did not monitor clinician use of the referral pads after the study finished. Future studies need to investigate whether clinicians continue to use this tool once the intervention period ends. Pairing this referral mechanism with supportive care screening tools could help facilitate patient-clinician discussions concerning supportive care needs of patients and families. This may lead to a more sustainable model by reducing time required by clinicians to assess supportive care needs while also empowering the patient to identify services of benefit to them. Furthermore, introducing electronic resources in the hospital setting would support a means to linking patients directly with CIS services, and should be considered.

This study has several strengths. The framework we proposed to implement a referral mechanism to community-based cancer support services in the hospital was developed in consultation with clinicians, ensuring the mechanism was appropriate in a clinical setting. Testing the intervention in two different hospitals shows the intervention is appropriate for both large and smaller hospitals. The monitoring of calls to CIS allowed us to determine the pattern of calls to this service from study sites before and after the intervention, as well as understanding trends in total calls to this service. However, several limitations also need to be acknowledged. Not all callers to CIS were asked about their treating hospital, which means the number of calls reported for each site might be an underestimation of the true call volume. Secondly, patients may have elected to contact CIS using email rather than telephone call. Information regarding treating hospital could not be obtained from email correspondence as most cases involved a single email being sent from each party. The average time between patients receiving the referral to participating in the phone interview was one month. While this was intended to allow time for the patient to act on the referral, this timeframe may have affected recall of the appointment with their clinician. Future studies may examine the possibility of conducting the interview closer to the referral process. Finally, we did not obtain the number of patients that did not receive the referral.

This study supports the feasibility, acceptability, and effectiveness of a novel method of referring cancer patients to community-based supportive care services. While it may seem logical to provide patients with information about community-based support services during their cancer treatment, our study suggests there are challenges inherent in this period that include information overload, needs being addressed by the health service and treatment needs dominating the patient's attention. Further work is needed to identify the care points when patients may be most receptive of a referral to CIS. 


\section{Compliance with ethical standards}

Conflict of interest statement: The authors declare that there are no conflicts of interest. The authors have full control of all primary data and agree to allow the journal to review the data if requested.

\section{Funding}

Funding for this study was provided by the Cancer Council Victoria (institutional funding). 


\section{References}

1. Dunn J, Lynch B, Rinaldis M, Pakenham K, McPherson L, Owen N, Leggett B, Newman B, Aitken J (2006) Dimensions of quality of life and psychosocial variables most salient to colorectal cancer patients. Psychooncology 15:20-30

2. Liekweg A, Eckhardt M, Taylor SC, Erdfelder E, Jaehde U (2005) Psychometric assessment and application of a questionnaire measuring patient: satisfaction with information on cancer treatment. Pharm World Sci 27 (2):96-103

3. Meneses KD, McNees P, Loerzel VW, Su X, Zhang Y, Hassey LA (2007) Transition from treatment to survivorship: effects of a psychoeducational intervention on quality of life in breast cancer survivors. Oncol Nurs Forum 34 (5):1007-1016. doi:10.1188/07.ONF.10071016

4. Griggs JJ, Sorbero ME, Mallinger JB, Quinn M, Waterman M, Brooks B, Yirinec B, Shields CG (2007) Vitality, mental health, and satisfaction with information after breast cancer.

Patient Educ Couns 66 (1):58-66. doi:10.1016/j.pec.2006.10.008

5. Clarke SA, Booth L, Velikova G, Hewison J (2006) Social support: gender differences in cancer patients in the United Kingdom. Cancer Nurs 29 (1):66-72

6. Turner J, Zapart S, Pedersen K, Rankin N, Luxford K, Fletcher J (2005) Clinical practice guidelines for the psychosocial care of adults with cancer. Psycho-Oncology 14 (3):159-173. doi:10.1002/pon.897

7. Harrison JD, Young JM, Price MA, Butow PN, Solomon MJ (2009) What are the unmet supportive care needs of people with cancer? A systematic review. Supportive Care in Cancer 17 (8):1117-1128. doi:10.1007/s00520-009-0615-5

8. Hasegawa T, Goto N, Matsumoto N, Sasaki Y, Ishiguro T, Kuzuya N, Sugiyama Y (2016) Prevalence of unmet needs and correlated factors in advanced-stage cancer patients receiving rehabilitation. Supportive Care in Cancer 24 (11):4761-4767. doi:10.1007/s00520016-3327-7

9. Jefford M, Black C, Grogan S, Yeoman G, White V, Akkerman D (2005) Information and support needs of callers to the Cancer Helpline, The Cancer Council Victoria. European Journal of Cancer Care 14 (2):113-123. doi:10.1111/j.1365-2354.2005.00505.x 10. Boltong A, Ledwick M, Babb K, Sutton C, Ugalde A (2017) Exploring the rationale, experience and impact of using Cancer Information and Support (CIS) services: an international qualitative study. Support Care Cancer 25 (4):1221-1228. doi:10.1007/s00520016-3513-7

11. Hutchison SD, Steginga SK, Dunn J (2006) The tiered model of psychosocial intervention in cancer: a community based approach. Psychooncology 15 (6):541-546. doi:10.1002/pon.973

12. Heckel L, Fennell KM, Mohebbi M, Byrnes M, Livingston PM (2017) Demographic characteristics, call details and psychosocial support needs of the family/friends of someone diagnosed with cancer who access Australian Cancer Council telephone information and support services. European Journal of Oncology Nursing 28:86-91. doi:10.1016/j.ejon.2017.03.007

13. Beesley VL, Janda M, Eakin EG, Auster JF, Chambers SK, Aitken JF, Dunn J, Battistutta D (2010) Gynecological cancer survivors and community support services: referral, awareness, utilization and satisfaction. Psychooncology 19 (1):54-61. doi:10.1002/pon.1528 14. Eakin EG, Strycker LA (2001) Awareness and barriers to use of cancer support and information resources by HMO patients with breast, prostate, or colon cancer: patient and provider perspectives. Psychooncology 10 (2):103-113

15. Kam LY, Knott VE, Wilson C, Chambers SK (2012) Using the theory of planned behavior to understand health professionals' attitudes and intentions to refer cancer patients for psychosocial support. Psychooncology 21 (3):316-323. doi:10.1002/pon.1897

16. Dilworth S, Higgins I, Parker V, Kelly B, Turner J (2014) Patient and health professional's perceived barriers to the delivery of psychosocial care to adults with cancer: a systematic review. Psychooncology 23 (6):601-612. doi:10.1002/pon.3474 
17. Broadstock MJ, Hill D (1997) Evaluation and impact of promotion of a cancer helpline to cancer patients through their specialists. Patient Education and Counseling 32 (3):141-146 18. Livingston PM, White VM, Hayman J, Maunsell E, Dunn SM, Hill D (2010) The psychological impact of a specialist referral and telephone intervention on male cancer patients: a randomised controlled trial. Psychooncology 19 (6):617-625.

doi:10.1002/pon.1609

19. O'Brien MA, Rogers S, Jamtvedt G, Oxman AD, Odgaard-Jensen J, Kristoffersen DT, Forsetlung L, Bainbridge D, Freemantle N, Davis D, Haynes RB, Harvey E (2007)

Educational outreach visits: effects on professional practice and health care outcomes. The Cochrane Library. doi:10.1002/14651858.CD000409.pub2

20. Akbari A, Mayhew A, Al-Alawi MA, Grimshaw J, Winkens R, Glidewell E, Pritchard C, Thomas R, Fraser C (2008) Interventions to improve outpatient referrals from primary care to secondary care. Cochrane Database of Systematic Reviews (4).

doi:10.1002/14651858.CD005471.pub2

21. Roila F (2004) Transferring scientific evidence to oncological practice: a trial on the impact of three different implementation strategies on antiemetic prescriptions. Support Care Cancer 12 (6):446-453. doi:10.1007/s00520-003-0553-6

22. Grimshaw JM, Shirran L, Thomas R, Mowatt G, Fraser C, Bero L, Grilli R, Harvey E, Oxman A, O'Brien MA (2001) Changing provider behavior: an overview of systematic reviews of interventions. Med Care 39 (8 Suppl 2):II2-45

23. Clinton-McHarg T, Paul C, Boyes A, Rose S, Vallentine P, O'Brien L (2014) Do cancer helplines deliver benefits to people affected by cancer? A systematic review. Patient Educ Couns 97 (3):302-309. doi:10.1016/j.pec.2014.09.004

24. Sheffer MA, Baker TB, Fraser DL, Adsit RT, McAfee TA, Fiore MC (2012) Fax referrals, academic detailing, and tobacco quitline use: a randomized trial. Am J Prev Med 42 (1):2128. doi:10.1016/j.amepre.2011.08.028

25. Kirst M, Schwartz R (2015) Promoting a smokers' quitline in Ontario, Canada: an evaluation of an academic detailing approach. Health Promot Int 30 (2):310-317. doi:10.1093/heapro/dat040

26. Campbell S, Pieters K, Mullen KA, Reece R, Reid RD (2011) Examining sustainability in a hospital setting: case of smoking cessation. Implement Sci 6:108. doi:10.1186/1748-59086-108 
Table 1 Clinicians' perceptions of the education session $(N=40)$

\begin{tabular}{lcc}
\hline Question & Mean (SD) & Median $^{\#}$ \\
\hline The information was interesting & $3.43(0.75)$ & 4 \\
The information was easy to understand & $3.45(0.68)$ & 4 \\
The information was useful to me & $3.45(0.68)$ & 4 \\
I learnt something new at this session & $3.50(0.75)$ & 4 \\
The speaker had good knowledge of the topics & $3.63(0.77)$ & 4 \\
I had opportunities to ask questions & $3.53(0.75)$ & 4 \\
Attending the session was worthwhile & $3.51(0.68)$ & 4 \\
\hline "range [1 - 4]: 1 = "Strongly disagree", 2 = "Disagree", 3 = "Agree", 4 = "Strongly agree".
\end{tabular}

Table 2 Clinicians' assessment of feasibility and acceptability of intervention at end of study period $(\mathrm{N}=40)$

\begin{tabular}{lc}
\hline Evaluation item & N/valid (\%) $^{\mathbf{*}}$ \\
\hline & \\
Feasibility & $29 / 33(88 \%)^{\#}$ \\
'Very easy' or 'Easy' to use referral pad during consultations & $37 / 40(93 \%)$ \\
Referral process can be incorporated in routine practice: 'Yes' & $25 / 35(71 \%)$ \\
Time taken to refer patient: '2-5 minutes' & \\
& \\
Barriers preventing referrals & $10 / 34(29 \%)$ \\
'Time' & $19 / 36(53 \%)$ \\
'Overlooked' & $11 / 34(32 \%)$ \\
'Patient condition' & $7 / 33(21 \%)$
\end{tabular}

Acceptability

'Very confident' or 'Confident' in CIS supporting patients $\quad 27 / 39(69 \%)^{\dagger}$ Continue to refer after study: 'Yes'

${ }^{7}$ percentages based on number of valid responses

\# 5 -point scale

${ }^{\dagger}$ 4-point scale

Table 3 Patients' reasons for not calling CIS ( $\mathrm{N}=45)$

\begin{tabular}{cl}
\hline Theme & Reasons \\
$\begin{array}{c}\text { Forgetting } \\
(\mathrm{N}=8)\end{array}$ & . forgot about the referral \\
$\begin{array}{c}\text { No need } \\
(\mathrm{N}=16)\end{array}$ & memory loss due to treatment-related side effects \\
Timing & services not needed at the time of the referral \\
$(\mathrm{N}=11)$ & - not the right time to receive the referral \\
Burden of information & other things deemed more important \\
$(\mathrm{N}=6)$ & - large volume of information received at diagnosis and treatment stages \\
\end{tabular}




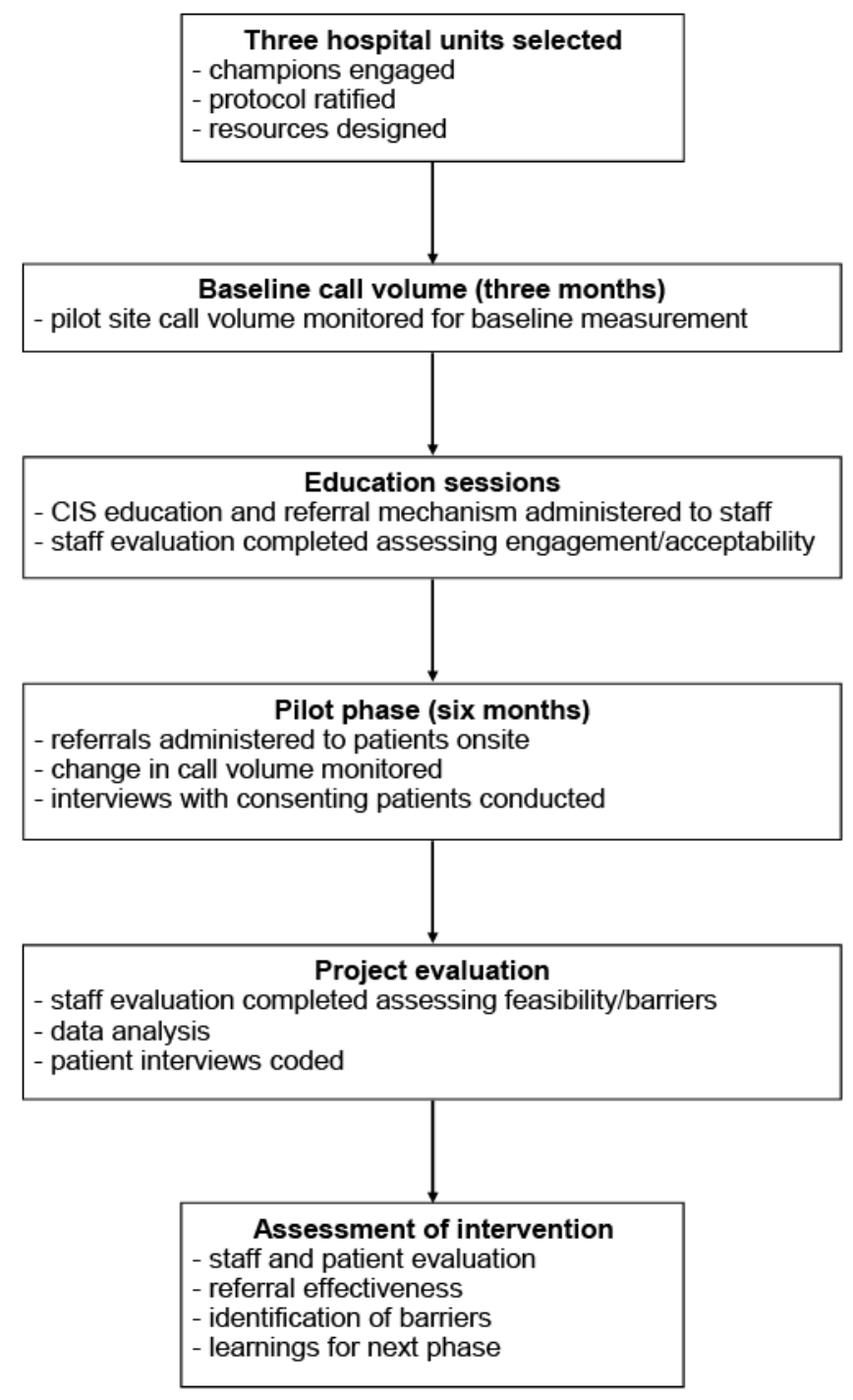

Figure 1 Study design. 


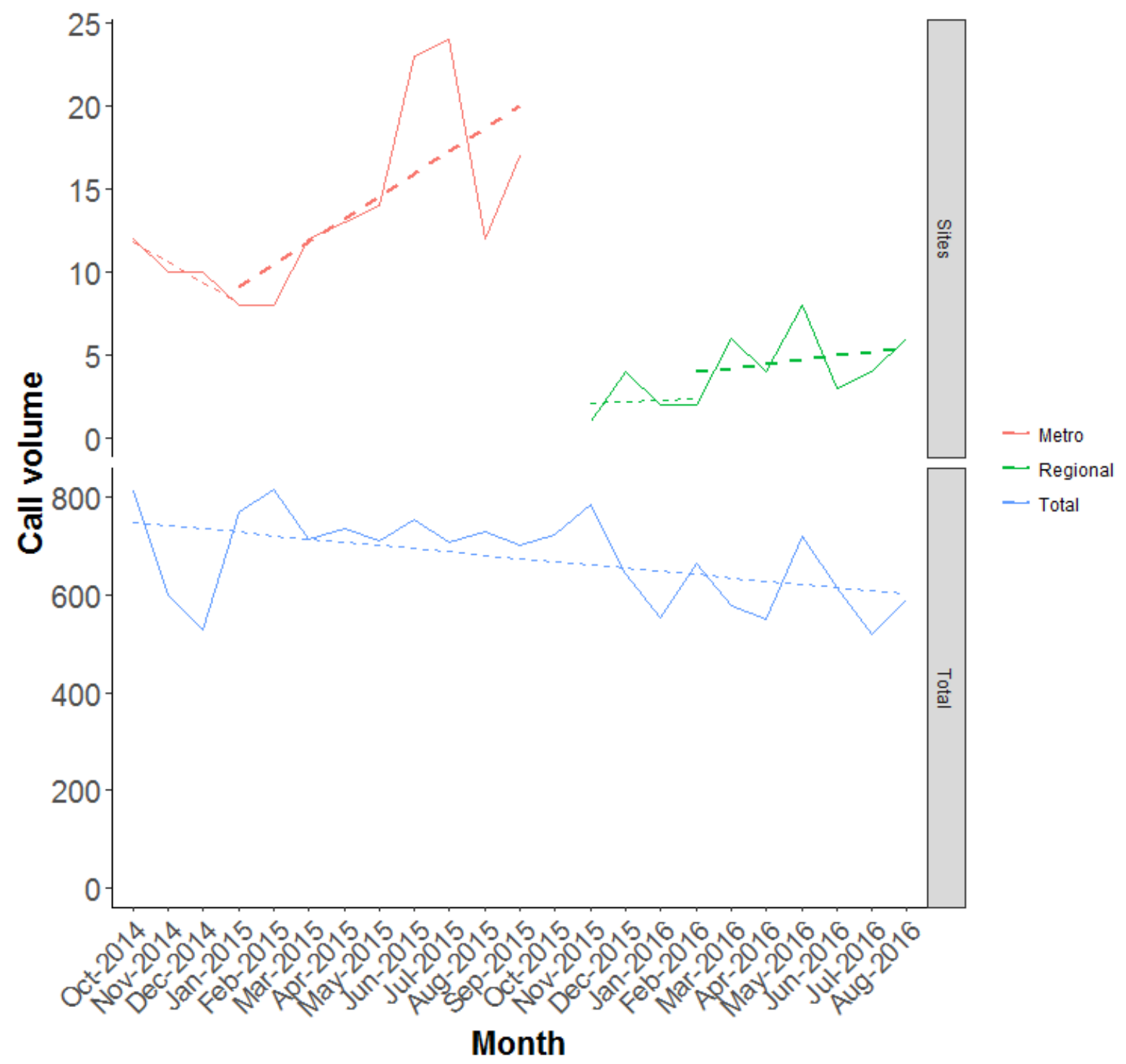

Figure 2 Calls to CIS Absolute call volume is shown for each study site including 3 months' baseline data plotted above total call volume to CIS from October 2014 to August 2016. Bold dashed linear regression lines indicate monthly call trend during the intervention at each site compared to baseline (thin dashed linear regression lines). The Metro (Metropolitan) site intervention period was longer than Regional site due to a staggered intervention rollout across units. The intervention was rolled out concurrently across units in the Regional site. 